\title{
əIntransitive Atmosphere Dynamics Leading to Persistent Hot-Dry or Cold-Wet European Summers
}

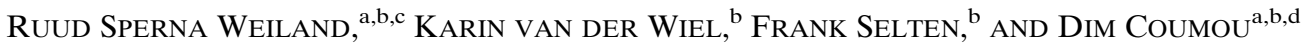

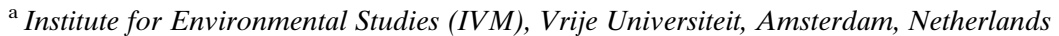 \\ ${ }^{\mathrm{b}}$ Royal Netherlands Meteorological Institute, De Bilt, Netherlands \\ ${ }^{\mathrm{c}}$ Rabobank, Utrecht, Netherlands \\ ${ }^{\mathrm{d}}$ Potsdam Institute for Climate Impact Research, Potsdam, Germany
}

(Manuscript received 7 December 2020, in final form 28 April 2021)

\begin{abstract}
Persistent hot-dry or cold-wet summer weather can have significant impacts on agriculture, health, and the environment. For northwestern Europe, these weather regimes are typically linked to, respectively, blocked or zonal jet stream states. The fundamental dynamics underlying these circulation states are still poorly understood. Edward Lorenz postulated that summer circulation may be either fully or almost intransitive, implying that part of the phase space (capturing circulation variability) cannot be reached within one specific summer. If true, this would have major implications for the predictability of summer weather and our understanding of the drivers of interannual variability of summer weather. Here, we test the two Lorenz hypotheses (i.e., fully or almost intransitive) for European summer circulation, capitalizing on a newly available very large ensemble (2000 years) of present-day climate data in the fully coupled global climate model EC-Earth. Using self-organizing maps, we quantify the phase space of summer circulation and the trajectories through phase space in unprecedented detail. We show that, based on Markov assumptions, the summer circulation is strongly dependent on its initial state in early summer with the atmospheric memory ranging from 28 days up to $\sim 45$ days. The memory is particularly long if the initial state is either a blocked or a zonal flow state. Furthermore, we identify two groups of summers that are characterized by distinctly different trajectories through phase space, and that prefer either a blocked or zonal circulation state, respectively. These results suggest that intransitivity is indeed a fundamental property of the atmosphere and an important driver of interannual variability.
\end{abstract}

KEYWORDS: Blocking; Dynamics; Jets; Summer/warm season; Climate classification/regimes; Interannual variability

\section{Introduction}

The Northern Hemisphere summer of 2018 was characterized by concurrent hot and dry extremes across North America, Europe, and Asia (Vogel et al. 2019). A large contributing factor to these extremes was a recurrent atmospheric circulation pattern (Kornhuber et al. 2019; Drouard et al. 2019; Li et al. 2020). In western Europe, hot-dry conditions extended from April to September, leading to one of the most severe droughts in terms of its impact on agriculture and the ecosystem (Buras et al. 2020; Philip et al. 2020; Vanderkelen et al. 2020; Beillouin et al. 2020). Model projections show a systematic increase in the persistence of summer weather, which might favor prolonged drought conditions, but also persistent precipitation extremes (Pfleiderer et al. 2019). In Europe, hotdry extremes are typically linked to a blocked circulation state (Simmonds 2018). In contrast to hot-dry summers, persistent cold-wet summers are typically caused by a zonally oriented midlatitude jet stream (Pasquier et al. 2019). One possible

\footnotetext{
D Denotes content that is immediately available upon publication as open access.
}

Corresponding author: Ruud Sperna Weiland, ruud.spernaweiland@ knmi.nl explanation of the persistence of these extremes could be that these circulation states reflect metastable equilibrium states of the large-scale flow, as initially suggested by Charney and DeVore (1979) and later by others (e.g., Branstator and Berner 2005; Sempf et al. 2007; Tantet et al. 2015). Nowadays, the presence of multiple regimes in the Northern Hemisphere atmosphere such as these equilibrium states is well established for winter circulation (Hannachi et al. 2017). However, a convincing dynamical explanation is still lacking, and the presence of distinct regimes is still debated for summer circulation (Ghil 2019). Motivated by the potential to improve seasonal predictability (Frame et al. 2011; Franzke and Woollings 2011; Franzke et al. 2020), and improving assessments of future climate risks associated with a changing circulation (Hannachi et al. 2017), we aim to contribute to the fundamental understanding of the atmospheric summer circulation over Europe and how it evolves into preferred flow dynamics.

The classical work by Edward Lorenz (Lorenz 1963) forms the basis of our current view on the atmosphere as a complex dynamical system. In a dynamical system, a function describes the evolution over time of a point in a phase space. At any time, the system is in a specific state of this phase space, and the evolution of the system through phase space is called a trajectory. An attractor is a set of states (i.e., a bounded region of the phase space) that neighboring states approach during the evolution of the dynamical system. A famous example of this is 
(a) Almost intransitivity

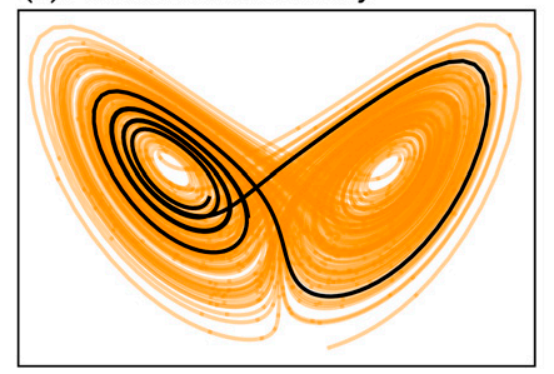

(b) Fully intransitive system with two distinct attractors

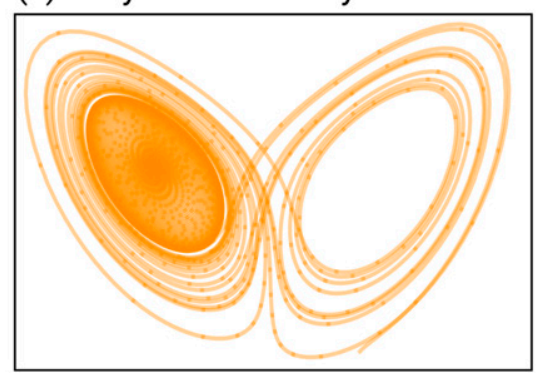

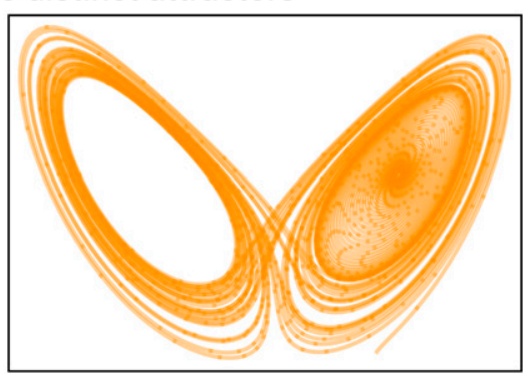

FIG. 1. Three different illustrative configurations of the classical Lorenz attractor, representing Lorenz's different hypotheses on the intransitive dynamics within a summer. (a) The black line is a representation of almost intransitivity; a single summer is too short to capture the whole phase space, hence dependence on the initial condition is strong. (b) Two attractors that have distinctly different preferred states of the phase space, illustrative of a fully intransitive system.

the Lorenz attractor (see Fig. 1a). Two fundamental properties that dynamical systems may display are chaos and intransitivity. Chaotic systems are extremely sensitive to the initial conditions and display no periodicity (i.e., recurrent behavior). At relatively short time scales the atmosphere behaves as a chaotic system-putting intrinsic limits to weather predictability (Lorenz 1963, 1982). An intransitive system is characterized by two or more mutually coexisting attractors. Which attractor will be traversed is determined by the initial state of the system. In contrast, the long-term dynamical characteristics of transitive systems are not affected by the initial state as there is only one attractor. In one of his papers, Lorenz suggests that "the atmosphere-ocean-earth system is unlikely to be intransitive" (Lorenz 1990, p. 378) on time scales longer than a season. However, Lorenz postulates that, if the seasons could be fixed, say at summer-with constant solar forcing - a specific dynamical regime could establish and persist indefinitely. In winter the atmosphere is more chaotic, since, due to the stronger solar heating contrast between equator and pole, the large-scale flow is more energetic with stronger instabilities making the flow more turbulent. The chaotic behavior in winter essentially randomizes the following summer's initial state, thus facilitating the transition between different summer attractors or different parts of the summer phase space. Chaos and intransitivity may therefore be a principal cause for interannual variability between summers. Whether the real atmosphere displays intransitive dynamics in summer is as yet unknown. Lorenz's study of intransitive summers was limited by his, by current standards, highly rudimentary model, since it consisted of only three equations describing the interaction of a single Rossby wave with a zonal flow component, which is a very crude dynamical model. In the current age of complex coupled climate models and increasing computing power, we have the tools to study intransitivity using more realistic data and with more advanced analysis algorithms.

\section{a. Two hypotheses: Almost and fully intransitive summers}

Lorenz offered two hypotheses (Lorenz 1968, 1990). The first hypothesis (Fig. 1a) defines almost intransitive systems that display characteristics of both transitive and intransitive systems. In almost intransitive systems there is only a single summer attractor determining the characteristics of the atmospheric circulation. The initial state (i.e., at which point in phase space the summer starts) therefore determines the trajectory through phase space throughout the remainder of the summer. Ultimately, the circulation statistics become independent of the initial conditions as the full phase space can be traversed, just as in a transitive system. However, on a shorter time scale, the circulation statistics can strongly depend on the initial condition, which thus resembles an intransitive system (Lorenz 1968). Lorenz hypothesized that the length of the summer is simply too short such that only a small part of the attractor is sampled within one summer, leading to almost intransitivity.

The reasoning behind this hypothesis is that in winter and spring the atmosphere is chaotic due to the weaker solar heating, and processes during this time determine the state of the atmosphere at the start of the summer. Figure 1a schematically illustrates the subsequent dynamics during summer, using the classical Lorenz attractor (Lorenz 1963) (used here solely for illustrative purposes; any realistic summer attractor will be much more complex). Suppose that the Lorenz attractor in Fig. 1a captures the phase space of circulation patterns, with the left wing of the attractor a hot-dry regime with a high blocking occurrence and the right wing to be a cold-wet regime with strong westerly flow. Almost intransitivity implies that, depending on early summer conditions, the summer trajectory is likely to remain in a particular part of the phase space (i.e., predominantly in hot-dry or predominantly cold-wet states). In theory, every state on the attractor has a climatological probability of being visited in a given summer if the trajectory is long enough such that the memory of the initial condition is lost. However, the summer season could well be too short for this to be the case before the heating distribution changes with the onset of autumn. To illustrate this, the black line represents one possible summer trajectory over the phase space. The system arrives at the summer attractor near the center of the left regime, and stays there for the majority of the summer.

Lorenz's second hypothesis (Lorenz 1990) assumes that instead of a single summer attractor, such as for an almost intransitive system, there are multiple attractors, each with their own trajectories, preferred states, and underlying dynamics. 
He identified two attractors in a simplified atmospheric circulation model: one with strong oscillations in the large-scale zonal winds and one with relatively weak variations in zonal wind. Conceptually this is similar to the metastable preferred states of the atmosphere as suggested by Charney and DeVore (1979). Figure 1b illustrates our view of the fully intransitive concept, again illustrated with the Lorenz attractor. The phase space and shape of the two attractors is similar in both figures but there is a clear preference for either the left wing (left panel of Fig. 1b) or right wing (right panel of Fig. 1b). Individual summers may therefore have markedly different regimes of behavior (Leith 1973) that perpetually influence trajectories through the phase space and possibly the preferred atmospheric flow until the onset of autumn, leading to distinctly different summers from year to year. Such a system is fully intransitive on a seasonal time scale, as there are multiple possible summer attractors that, for perpetual summer solar forcing, would structurally alter the long-term statistics of the system.

\section{b. Research question and experiment design}

For the real atmosphere, with only on the order of $50 \mathrm{ob}-$ served seasons, we cannot test the intransitivity hypothesis due to a lack of data. However, Lorenz speculated in 1968 that in advanced climate models (as we have today), intransitivity may become the rule rather than the exception, since these models take into account the complex interactions within the atmosphere-ocean-land system, thus introducing more memory and low-frequency variability into the atmosphere through heat and water exchanges with the underlying ocean and land (Hannachi et al. 2017). With the current computing power and state-of-the-art climate models, we can generate sufficient amounts of more realistic data to revisit this old question. Our study focuses on Europe, also motivated by the extremely persistent hot-dry conditions in 2018 (Kornhuber et al. 2019). From 2000 years of present-day EC-Earth climate simulations, we select the months July and August to define a discrete phase space of summer circulation patterns (states) over Europe (section 4). Within this phase space, we study the dynamics of summer trajectories to test Lorenz's two hypotheses. To test the first hypothesis of almost intransitivity, we analyze the dependence of the summer trajectories on the initial circulation state (section 5). We train a Markov model on the 2000 summers and use it to generate a very large number (200000) of Markov summers. In these Markov summers, we quantify after how many days since the start of the summer the probability of summer states loses its dependence on the initial state. We test the second hypothesis of full intransitivity with a novel iterative clustering approach that divides the 2000 EC-Earth summers into two groups that are characterized by distinctly different dynamics (section 6). These two groups represent two different attractors of a fully intransitive system.

\section{Data}

We used simulated data from the EC-Earth model to study intransitivity in the European summer circulation. EC-Earth is a coupled global climate model with atmospheric, oceanic, land, and sea ice components. We used EC-Earth version 2.3 to simulate a large ensemble of present-day climate (van der Wiel et al. 2019). EC-Earth V2.3 is the official CMIP5 version and it has only minor differences compared to V2.2 as described in Hazeleger et al. (2012), such as the prescribed CMIP5 aerosol forcing. The model has a horizontal atmospheric resolution of approximately $100 \mathrm{~km}$ (spectral truncation T159) and contains 62 atmospheric layers, with 20 layers above $200 \mathrm{hPa}$, that partly resolve the stratosphere. Version 2.3 was the most recent official version of the model at the time this ensemble was generated.

Our aim is to have a large ensemble of fully coupled climate simulations with constant forcing and an absolute global mean temperature that is close to today's. To do so, we select from 16 transient climate runs over the period 1860-2100 (with historic forcings plus the RCP8.5 scenario after 2005), the 5-yr period when the absolute global-mean surface temperature corresponded to that of the reference period 2011-15 in the real world $\left(14.52^{\circ} \mathrm{C}\right)$. Since EC-Earth has a cold bias, this global mean temperature is only reached in the model years 2035-39 (i.e., at a higher $\mathrm{CO}_{2}$ forcing). We emphasize that since we select this period from the 16 different transient climate runs, the ocean, sea ice, and soil moisture states are essentially randomly sampled. Of course, the modeled climate states are also not correlated in any way with the state of these components in the real-world period 2011-15 (except for the same absolute global mean temperature). The model states on 1 January 2035 of each of the 16 transient runs were used as an initial condition for an ensemble of 25 fully coupled runs of 5 years each, totaling $16 \times 25 \times 5=2000$ years of daily global data representative of the present-day climate under constant forcing. The 25 members differ in the random seed of the stochastic physics parameterization, leading to a rapid divergence of the 25 trajectories due to the chaotic nature of the atmosphere. Additional information on the experimental setup is given in van der Wiel et al. (2019).

There is no correlation between the 16 ensembles, since each starts from a very different initial state. Within each ensemble, small correlations between the 25 members persist beyond the chaotic prediction horizon of the atmosphere of a couple of weeks, since each starts from the same ocean-sea ice-land initial condition. For our study on the European summer circulation this is likely not an issue, since the atmospheric trajectories are well diverged during the six months prior to the first summer period that we evaluate. The influence of the small correlation of the land-ocean-sea ice state still present after six months between the 25 members on the summer circulation state is likely very small, and most certainly so for the summers of the second to fifth years. The state of the atmosphere at the start of each summer is therefore sampled virtually randomly on the summer attractor. This is confirmed by checking that the analysis of the European summer circulation dynamics in this study did not depend on the specific year in the 5 -yr runs. With this in mind and the fact that we use 16 uncorrelated initial conditions for the ocean-land-sea ice components, we conclude that our dataset yields a dynamically diverse set of summers that is representative of the summer climate attractor and suitable for studying intransitivity. 
Since we are interested in the synoptic circulation over Europe, we use the geopotential height field at the $500-\mathrm{hPa}$ level over the region $15^{\circ} \mathrm{W}-40^{\circ} \mathrm{E}, 30^{\circ}-70^{\circ} \mathrm{N}$. We calculated daily geopotential height anomalies for each grid point in this region. To reduce the influence of small-scale weather systems on the variability of the synoptic circulation, a low-pass filter is applied to the daily data, which removes high frequencies of variations shorter than three days but does not affect the structure of the jet stream and other large-scale circulation features. The first analysis showed that the circulation in the month of June differs significantly from the circulation in July and August, as the jets and storm tracks are still propagating northward during this month (Yeh 1959; Lu and Schneider 2017). For this reason we limit our analysis to the "high summer" months July and August that are dynamically similar. Therefore the final dataset contains $2000 \times 62=124000$ days. We use principal component analysis to reduce the dimensionality of the data (Ross et al. 2008). With a relatively small spatial domain and with the reduced variability by low-pass filtering, 20 principal components are sufficient to retain $99 \%$ of the variance.

\section{Methods}

\section{a. Defining the phase space of circulation regimes with self- organizing maps}

To assess the phase space of the atmosphere in summer over Europe, we discretize all states (i.e., individual days) into clusters. We use a self-organizing map (SOM) to classify circulation regimes on a phase map. A SOM or Kohonen map (Kohonen 1990) is a neural network architecture based on competitive learning. This forces neurons to self-organize to the shape of the data in its high-dimensional space. On top of this, a SOM also forms itself to a topographic map, which means that the spatial location of a node on the map corresponds to a feature from the input vector space. This has as a consequence that modes that closely resemble each other are also close together on the map, such that the map is topologically ordered. The chosen structure of the map is generally a one-dimensional line or a two-dimensional lattice of interlinked nodes. This allows for an easily interpretable visualization of the topographic map. When we apply a twodimensional SOM of size $15 \times 15$ to our atmospheric circulation data, we obtain a detailed phase space of circulation patterns with states that share features located close to each other, and states that are distinctly different located farther away from each other. This level of detail is needed to study the trajectories through the phase space accurately. This would not be possible in a more classical SOM analysis with a much smaller number of clusters as the jumps between neighboring states would be too large. In this research, we fit a torus SOM, similar to Ohba et al. (2016), which differs from a normal SOM in that the edges of the lattice are connected and hence the resulting map is continuous. Since trajectories through a phase space are continuous it is appropriate that the phase space itself is continuous too, in order to represent the trajectories in the most realistic way. One can envision the continuous phase space as a spherical surface, projected onto a square grid.
We use the discrete SOM phase space to compute the transition probabilities between states, based on the daily phase space states of all 2000 EC-Earth summers. For each state in the $15 \times 15$ phase space, we estimate the transition probabilities to every other state. One summer trajectory is 62 days, corresponding to July and August. To compute the $i$-day transition probabilities, we count how many times in the 2000 trajectories the state changes in $i$ days from one particular SOM state to any other SOM state, and we repeat this for every SOM state. The result is $15 \times 15=225$ matrices of size $15 \times 15$ with counts of how many times each transition has occurred. Next, each matrix is divided by the total number of transitions from that state respectively, to make the sum of all probabilities in each matrix equal to 1 . In the remainder of this paper, we refer to the transition matrix as the complete set of these 225 matrices.

\section{b. Testing almost intransitivity with Markov chains}

To test for almost intransitivity, we analyze ensembles of trajectories over the phase space of circulation patterns starting from every initial state in our phase space, and analyze for how long the ensembles remember their initial condition. Since the sample of 2000 EC-Earth summers is not large enough for getting robust results in this analysis, we perform an experiment in which we assume that the atmospheric circulation behaves according to a memoryless Markov process. Effectively this assumes that every summer behaves according to the same set of underlying dynamics, and that there is no memory from past states/days (i.e., no memory from days directly before the current day, only the current state matters). Given these assumptions, we can use the climatological oneday-ahead transition probabilities from a given state to all other states in order to simulate large ensembles of Markov summer trajectories of 62 days through phase space. We do this by starting at a chosen state and sequentially sampling transitions according to these probabilities.

The main advantage of using the Markov process compared to a fully coupled global climate model is that it is a computationally cheap method. This removes the restriction that we can only run a limited number of simulations, ensuring a large enough sample of trajectories for our analysis. A disadvantage is that in reality the atmospheric circulation is unlikely to resemble a Markov process, due to processes influencing atmospheric circulation on time scales longer than a day, such as sea surface temperatures, soil moisture, and solar heating, that build up over time. (Lorenz 1990; Kingston et al. 2015; Ionita et al. 2012; Fischer et al. 2007; García-Herrera et al. 2010). Since trajectories are sampled using the one-day-ahead transition probabilities, these longer-term processes only implicitly influence the Markov trajectories. On relatively short time scales (less than 3 days), this makes the Markov process more diffusive. On longer time scales, however, the Markov trajectories can show too little dispersion over the phase space, as compared to EC-Earth. On the one hand, the Markov trajectories can in theory traverse the phase space faster, since they do not have memory of previous states and are therefore less restricted in terms of the possible directions they can evolve into. After all, two subsequent one-day transitions sampled 
with the Markov model may not necessarily be observed in the EC-Earth trajectories as those are restricted by the memory of past states. On the other hand, the EC-Earth trajectories are more likely to diffuse the longer it has been in a specific state, due to the chaotic and transient nature of the atmospheric circulation. In Markov trajectories this tendency is not present as we always sample from the same transition matrix, which may result in more persistent Markov trajectories. These two competing effects make it challenging to determine whether memory effects, and thus the potential for almost intransitivity, are more or less pronounced in EC-Earth as compared to Markov trajectories. By design the Markov model can reproduce the EC-Earth trajectories, since the transition probabilities are estimated on the 2000 EC-Earth trajectories, but whether all potential Markov trajectories can in theory be reproduced by EC-Earth is unlikely. Note that in the Markov transition matrix it is possible to go from any state to any other state within a finite number of transitions, but not all transition probabilities are greater than zero (as we will show later on). This means that Markov trajectories cannot get stuck in a specific region of the phase space because eventually the Markov model will sample a transition to another part of the phase space.

To estimate the probability of states in dependence on the initial SOM state of the summer with good accuracy, we need about 200000 Markov trajectories of length 62 (corresponding to July and August) from each initial state. Next, for each day $n$ since the start of the summer with $n=1, \ldots, 62$, we calculate the probability distribution over states at that point in time. Hence, we get 62 probability distributions per initial state, where each probability distribution is based on 200,000 daily states. These are to be compared to the EC-Earth climatological probability distribution, which is based on $62 \times 2000=$ 124000 days. We compare these probability distributions for each day to the climatological probability distribution from the EC-Earth summers (Fig. 3) with the two-sample KolmogorovSmirnov test with a significance level of 0.05 (Kolmogorov 1933). The distribution of the 200000 trajectories initially depends on the initial condition by design, but the farther away in time from the start of the summer, the weaker the signal of the initial state and the more strongly the distribution will resemble the climatological probability distribution. Thus, after a certain number of days the Markov model has lost all memory of the initial state. If it takes most of the summer to lose the dependence on the initial state, we take this as a strong indication that the EC-Earth atmosphere is almost intransitive as well. We estimate this memory horizon for every possible initial state.

One could argue that it is more rational to use the three-dayahead transition probabilities instead of the one-day-ahead transition probabilities, since processes with time scales shorter than three days have been filtered out. We therefore also performed the same experiment with trajectories of 21 sequentially sampled 3-day transitions, since this is equivalent to trajectories with a time horizon of $3 \times 21=63$ days. This sensitivity analysis showed that there are no significant differences in estimated memory horizon between using the one-dayahead transition probabilities and using the three-day-ahead transition probabilities.

\section{c. Testing full intransitivity with a custom clustering method}

Assuming that the transition matrix reasonably captures the underlying dynamics, like we did in the previous experiment, then a fully intransitive system has multiple transition matrices (i.e., attractors), each with its own trajectories and preferred states. We developed a clustering algorithm capable of detecting such groups of trajectories and corresponding transition matrices from data. The algorithm is inspired by $k$-means clustering (Jain 2010). However, instead of computing the distances between observations and a cluster mean, our algorithm computes how well a summer trajectory (the observation) fits a cluster's transition matrix. Building on our results from the previous experiment (section 5), the initial clustering is semi-random and separates the 2000 EC-Earth summers in two groups that are farthest apart in the phase space. The clustering algorithm then iterates over two steps. The first step consists of reassigning trajectories to the group whose transition matrix fits the trajectory best. In the second step, once the best-fitting group for all trajectories has been evaluated, the three-day-ahead transition matrices are recomputed. Since the transition matrices are now different as compared to the previous iteration, the algorithm repeats these steps until there are no reassignments in the first step anymore. At this point, the algorithm has converged and terminates. The interplay between the varying group compositions and the varying transition matrices ensures that the transition matrix in each group converges to its own characteristic dynamics. To assess whether the algorithm consistently converges to the same solution, we run the algorithm 500 times while varying the initial grouping between runs. A more detailed description of the algorithm is given in the appendix.

\section{Phase space of circulation regimes}

The phase space of circulation regimes must adequately represent the variability of the system and therefore we fit a SOM of size $15 \times 15$ to the geopotential height data. Other map sizes were evaluated, but this size strikes a good balance between the amount of detail that is preserved in the phase space and the robustness of the results [since each state contains 124000 days $/(15 \times 15) \approx 550$ days on average]. The SOM phase space of the EC-Earth summer circulation is shown in Fig. 2. In the region stretching from I10 to N14 (red colors in Fig. 2), we see blocking-type circulation with anomalous high pressure over northern Europe (Fig. 2d). In contrast to this, the region A1:G6 (blue colors in Fig. 2) shows a strong zonal southerly shifted jet stream with lower than average geopotential height over northern Europe (Fig. 2c). Note that in terms of topological distance in phase space, these two regions are farthest away from each other, implying that these patterns have the least similarity. In the space in between the two regions, we see different type of states including northerly zonal flow in E9:H14, a weak zonal flow in H5:L8, and a split jet stream over the continent in H1:J4 (Fig. 2b). Last, A10:D14 shows a wavy jet stream. The general structure of the SOM phase space was consistent across multiple SOM runs with different parameters, indicating that the SOM clustering is not 
(a) Phase space of summer circulation

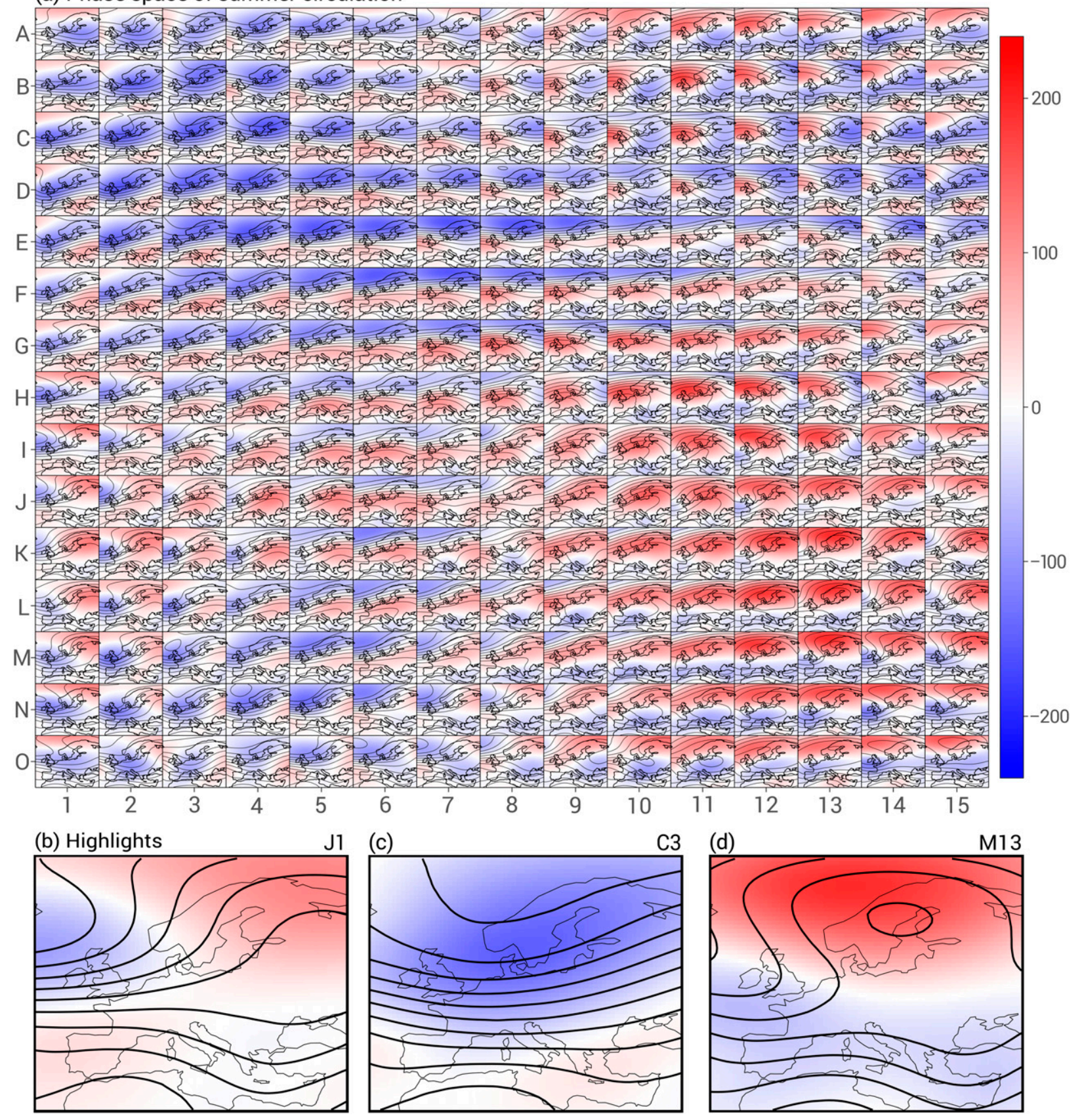

FIG. 2. Average geopotential height anomaly $(\mathrm{m})$ in colors and corresponding geopotential height $(\mathrm{m})$ in contour lines per node on the $15 \times 15 \mathrm{SOM}$, showing the phase space of European summer circulation patterns (July-August) in the EC-Earth model. Both the horizontal and vertical edges are connected to form a torus-shaped phase space. Black lines show contours of equal geopotential height at an interval of $50 \mathrm{~m}$. (b)-(d) Different states of the phase space.

sensitive to the specific parameter settings. We tested different sets of SOM sizes, which gave qualitatively the same results, and found the $15 \times 15$ SOM to be the best balance between details of the flow patterns versus sufficient sampling of individual states.

Figure 3 shows the climatological probability distribution over the states in the phase space. This heat map is smoothed by taking the average value over a $3 \times 3$ box around each state to emphasize the larger structures. The blocking region has the lowest probability density. The cumulative probability over all blocking states (within the cyan-colored outline from Fig. 3 ) is $\sim 0.18$, indicating that blocking occurs in less than $20 \%$ of summer days, which is in line with other analyses in literature 
Climatological probability distribution

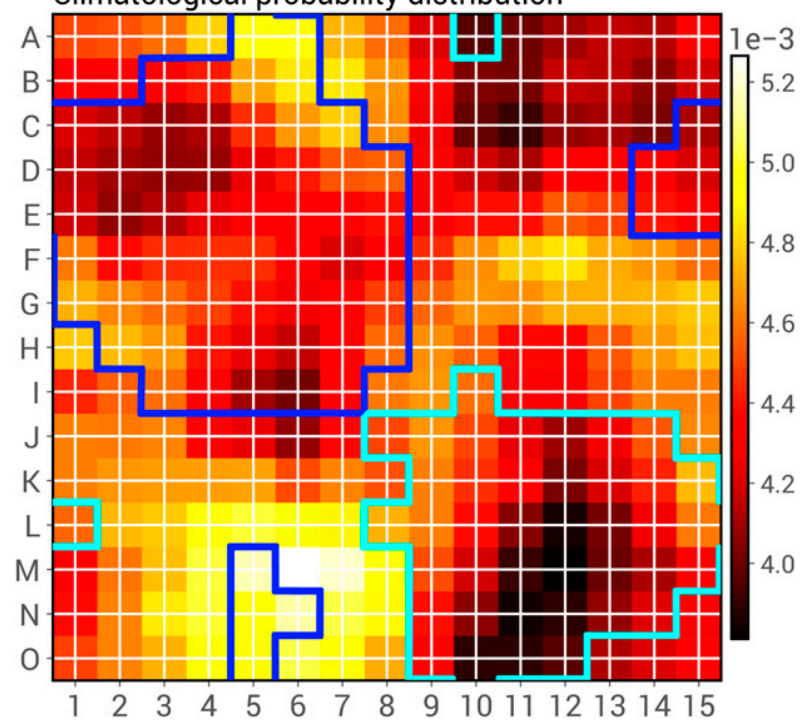

FIG. 3. Climatological probability distribution over all states in the phase space (as in Fig. 2), smoothed with a $3 \times 3$ filter. Lighter colors refer to relatively many days in this state and dark colors to relatively few days. Values sum up to 1 . Outlined regions indicate the southerly jet (dark blue) and blocking (cyan) region where the atmospheric memory (as defined in Fig. 5) is larger than 36.5 days.

on blocking occurrence (Cassou et al. 2005). The probability of the southerly jet region is higher than that of the blocking region, but still relatively low as compared to some other parts of the phase space. The cumulative probability over all southerly jet states (within the blue outline from Fig. 3 ) is $\sim 0.29$, but note that this region contains more individual states (66) than the blocking region (42). Nearly half of the probability mass is thus covered by the larger blocking and southerly jet regions, and the other half lies in between those regions.

Figure 4 shows the three-day-ahead transition probabilities for the three highlighted states in Figs. 2b-d. For all these states, the circulation is fairly persistent on a 3-day time scale, since the bulk of the transition probabilities is in or directly surrounding the current state. However, we also see a tendency to move into specific directions of phase space. The analyses show that in our sample the system cannot jump within three days from a blocked state into a southerly jet state or vice versa (gray regions in Fig. 4). So, in order to get there, the system first needs to traverse through the phase space in between these opposites.

\section{Dependence on initial state of circulation}

Following Lorenz's first hypothesis, we assess almost intransitivity by analyzing the dependence of each summer on its initial state. We define each state in the phase space as an initial state, and study the trajectories of Markov summers over the phase space starting from this initial state, as we described in section $3 \mathrm{~b}$. This allows us to analyze how long the signal of the initial state remains present throughout a summer.

Figure 5 shows the result of the Markov chain experiment we described in section $3 \mathrm{~b}$. The memory of the initial state is still significant up to 28-48 days, depending on the starting position. The southerly jet region (blue outline) and to a lesser extent the blocking region (cyan outline) stand out, with the longest memory ( $\sim 45$ days and 40 days respectively). Note that in these regions the atmospheric memory becomes close to the duration of our sampled summers (62 days). This result suggests that the initial state influences the probability of summer circulation states for the majority of the summer (July-August).

Figure 6 further illustrates how the initial state influences the probability distribution during the summer, specifically at day 20 and at day 35 . At day 20 the probability distribution is much higher around the initial state than at day 35. Furthermore, in Fig. 6a there is more diffusion over the phase space than in Figs. $6 \mathrm{~b}$ and $6 \mathrm{c}$. Note that at day 35 , the signal of the split jet stream initial state (J1) is already lost (Figs. 6d and 3 for comparison); for an initial state of southerly jet and blocking, this is not yet the case (Figs. 6e and 6f, respectively). Here, the probability mass is still much higher around the initial state than in other parts of the phase space, for both the southerly jet and blocking initial state. This indicates that both these (a) Transition probabilities

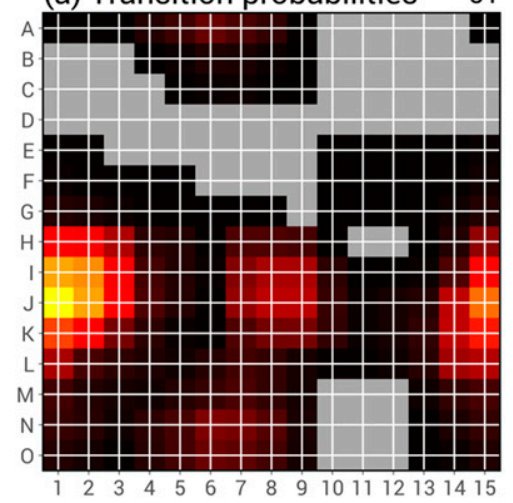

(b)

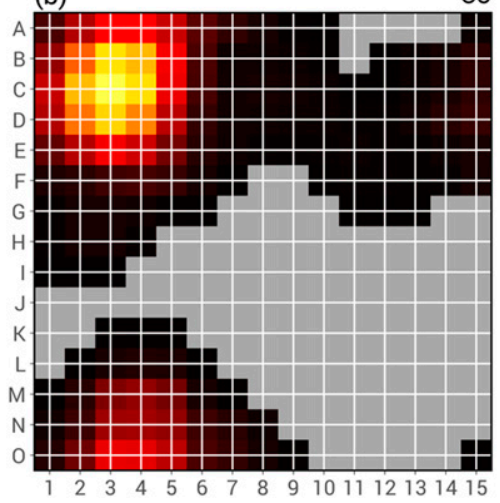

(c)

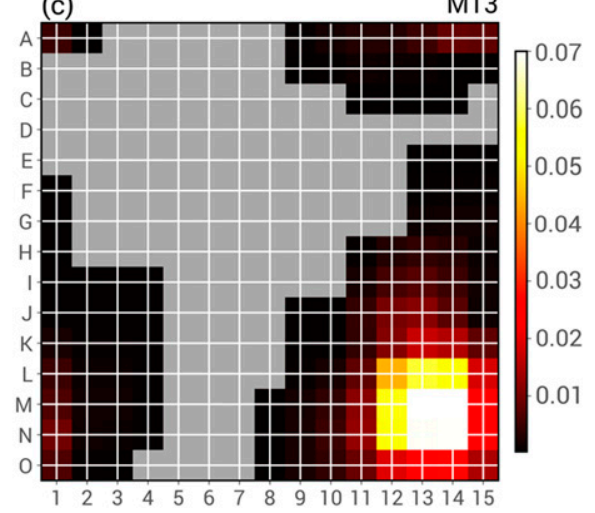

FIG. 4. Climatological 3-day transition probabilities in three highlighted states of the phase space (as in Fig. 2), smoothed with a $3 \times 3$ filter. In each matrix, the probabilities sum to 1 , and a probability of zero is given a gray color. 
Length of memory of initial state

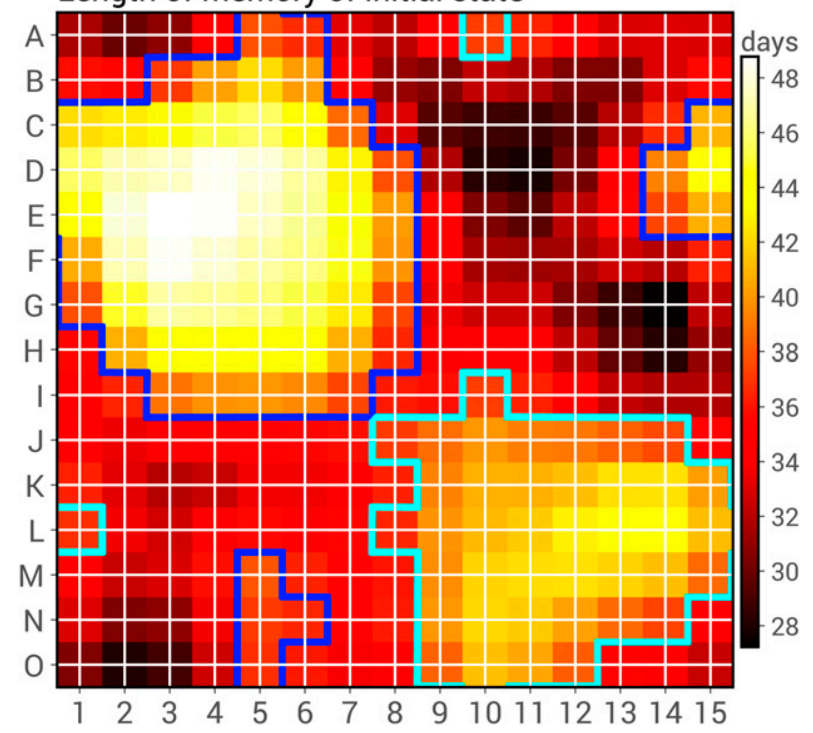

FIG. 5. Length of atmospheric memory of each initial state in number of days, smoothed with a $3 \times 3$ filter. This is defined to be the moment the distribution over the phase space of Markov sampled summers starting from the same initial state resembles the climatology as in Fig. 3 at a statistically significant level. Outlined regions indicate the southerly jet (dark blue) and blocking (cyan) region where the atmospheric memory is larger than 36.5 days.

circulation patterns are very persistent or recurrent and also that they tend to exclude each other to a high degree over the course of the summer. This persistence of the southerly jet and blocking region is also illustrated in Fig. 4, which shows that 3-day transitions in these regions are much less diffusive over the phase space (Figs. 4b,c) than the 3-day transitions outside of these regions (Fig. 4a). The tendency for mutual exclusion of the southerly jet and blocking regions is not surprising from a topological perspective. The two regions are both on different sides of the phase space in terms of Euclidean distance, and therefore well separated. Therefore it makes at least intuitively sense that when starting in one of these phase regions, it takes a longer time to reach the other.

Our Markov model experiment shows that summer circulation is not forever dependent on its initial condition, since the signal eventually diminishes. However, the results support the idea of almost intransitivity, since the estimated lower bound for the memory of the atmosphere's initial state is long, especially for the blocking and southerly jet states. This makes it unlikely to have summers that have a large presence in both the southerly jet region and in the blocking region, which we find indeed to be the case (Fig. 7). Here we separate the phase space based on a memory length of 36.5 days or more, which results in two separate regions in phase space: a region of southerly jet states and a region of blocking states. The exact outlines of the resulting regions are given in Fig. 5. We see that most of the 2000 EC-Earth summers (i.e., not based on the Markov model) have an above average number of days in either the blocking region $(33.0 \%$, Fig. 7$)$ or in the southerly jet region $(37.4 \%)$.
Only a small group of summers experienced both regimes with a higher than climatological probability (8.9\%). Finally, the summers in the bottom left quadrant of the scatterplot $(20.7 \%)$ have a below average presence in both regions.

To illustrate the predictive power of the initial state for the rest of the summer circulation, we look at the EC-Earth summer trajectories. If an EC-Earth summer starts in the southerly jet region on 1 July, the probability of being in the blocking region that summer is 0.15 , which is a relative decrease of $17 \%$ compared to the climatological blocking probability of 0.18 (within the cyan colored outline in Fig. 3). When starting in the southerly jet region, the probability of being in the southerly jet region is 0.36 , which is a relative increase of $24 \%$ compared to climatological probability of 0.29 (within the blue-colored outline in Fig. 3). On the other hand, if a summer starts in the blocking region on 1 July, the probability of also traversing the southerly jet region that summer is 0.23 , which is a relative decrease of $21 \%$ compared to the climatological probability (0.29). The blocking probability for summers starting in the blocking region is equal to 0.26 (relative increase of $44 \%$ ). The changes in probability are moderate, but this is at least partly due to the relatively large space covered by both regions. States at the edges of these regions less resemble southerly jet or blocking states respectively than states at the center of the regions. The latter have longer memory, and thus the changes in probability (as presented above) will be larger when starting in the center of the blocking or southerly jet region. These numbers illustrate that blocking and southerly jet circulation patterns are not strictly exclusive, but likely in some form dependent on each other. For example, recently it has been shown that blocking is likely to be more persistent in an $\mathrm{NAO}+$ event ( $\mathrm{Li}$ et al. 2020). This indicates that a strong jet stream (such as in the southerly jet region) and blocking events are not completely unrelated, but may both influence how summer trajectories will develop, a notion that we ignored in our Markov experiment. Unfortunately our spatial domain does not cover a large enough part of the North Atlantic to fully recognize the NAO phase in each state and therefore we cannot test this hypothesis specifically, but in the next section we do study differences in the underlying dynamics of the trajectories through the phase space in more detail.

\section{Separating summers based on their underlying dynamics}

We showed, assuming that all summers behave according to the same set of underlying dynamical rules (i.e., the same Markov transition probabilities), that the memory is relatively long. This supports the idea of almost intransitivity, as it shows that in a single summer it is unlikely that all regions of the phase space are visited with a climatological probability before the onset of autumn. Moreover, when the initial state lies in the southerly jet or the blocking region of the phase space, these states tend to be the dominant circulation pattern for the majority of the summer. In using the Markov transition probabilities for sampling trajectories, however, we assume the trajectories are not influenced by any forcing from the boundary conditions of the atmosphere, other than those 
(a) Distributions at day 20

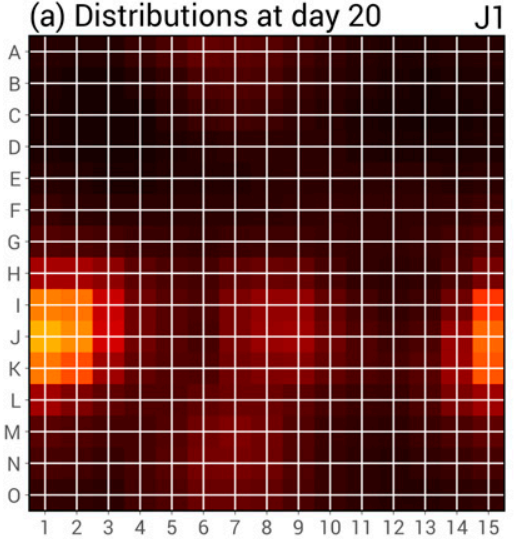

(d) Distributions at day 35

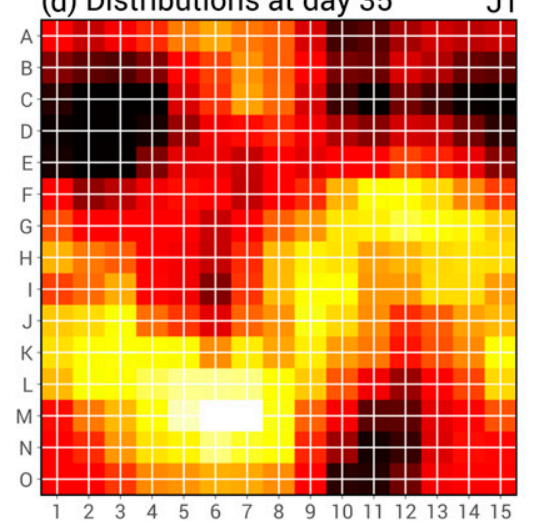

(b)

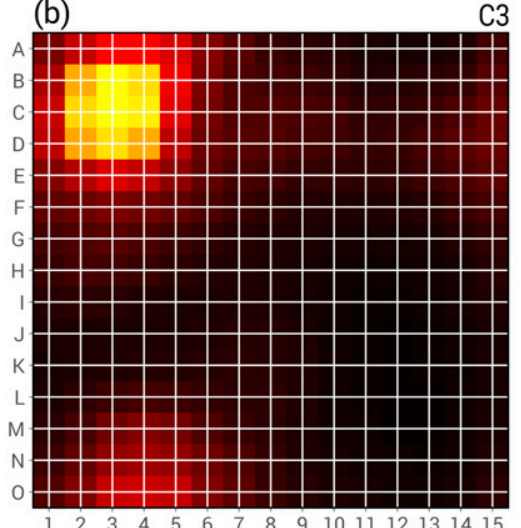

(e)

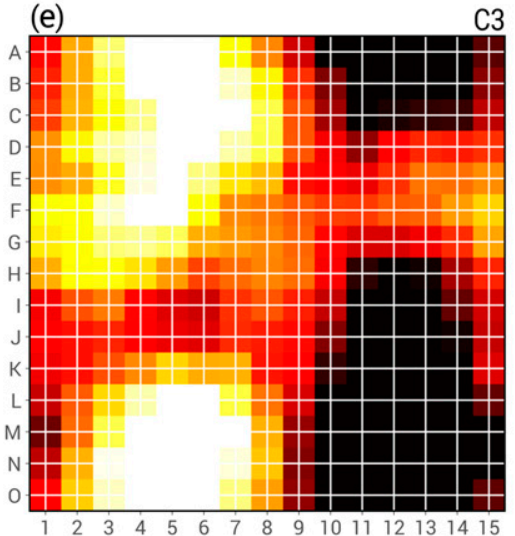

C3
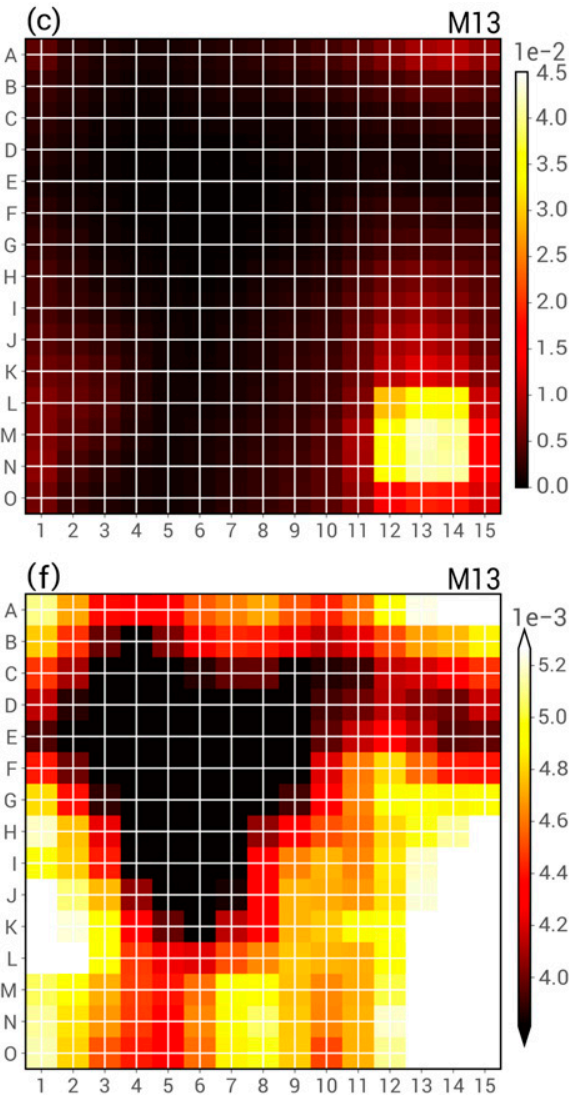

FIG. 6. Distributions over the phase space at (a)-(c) day 20 and (d)-(f) day 35 of 200000 sampled Markov summers, each starting in one of the three highlighted states of the phase space (Fig. 2), smoothed with a $3 \times 3$ filter. The color scale differs between (a)-(c) and (d)-(f); the scale in (d)-(f) corresponds to Fig. 3 for direct comparison.

that are implicitly captured in the transition probabilities. However, in the real climate system the circulation is conditional on the slowly varying boundary conditions (Lorenz 1976, 1990; Della-Marta et al. 2007; Ionita et al. 2012; Fischer et al. 2007; García-Herrera et al. 2010), which together with chaotic atmosphere dynamics determine the summer initial condition and which attractor the summer trajectory follows. As such, individual summers may behave according to different underlying dynamics. In our second experiment, following Lorenz's (1990) theory of a fully intransitive system, we therefore hypothesize that individual summers lie on different attractors (Fig. 1b).

We test this hypothesis by separating the 2000 modeled ECEarth summers into two groups that are dynamically most dissimilar. Although in principle there could be more attractors and associated sets of underlying dynamics, for simplicity we assume that two attractors exist, which is in line with both Lorenz's (1990) findings and the blocking versus zonal preferred states in Charney and DeVore (1979) and Charney et al. (1981). As in the previous experiment, we assume that the underlying dynamics of summer trajectories are adequately captured by the transition matrices, and thus each group of summers has its own transition matrix based on the summer trajectories within that group. To separate summers into two groups with distinct transition matrices, we use our custom clustering algorithm which is described in section $3 \mathrm{c}$ and covered in detail in the appendix.

The clustering is consistent over the 500 performed runs with varying initial groups. One summer group consistently has a clear preference for the southerly jet region in the phase space and the other group has a clear preference for the blocking region. Therefore, from this point onward, we denote the two groups as the group of southerly jet summers and the group of blocking summers. The final clustering is obtained by assigning each summer to the group it was a part of in the majority of the 500 runs. The result is that 1025 summers belong to the southerly jet summer group and 975 summers belong to the blocking summer group. This confirms our reasoning that the summers tend to prefer only one of two regions and that these regions tend to exclude each other during a given summer. Figure 8 shows the probability distributions over the phase space of these two groups. The preferred regions are easily distinguishable. In the southerly jet summer group (Fig. 8a), the probability is almost 3 times higher in the southerly jet region (probability of 0.35 ) than in the blocking region (probability of 0.13). In the blocking summer group (Fig. 8b), the southerly jet region has a probability of 0.23 and the blocking region has a probability of 0.24 . This contrast is less 


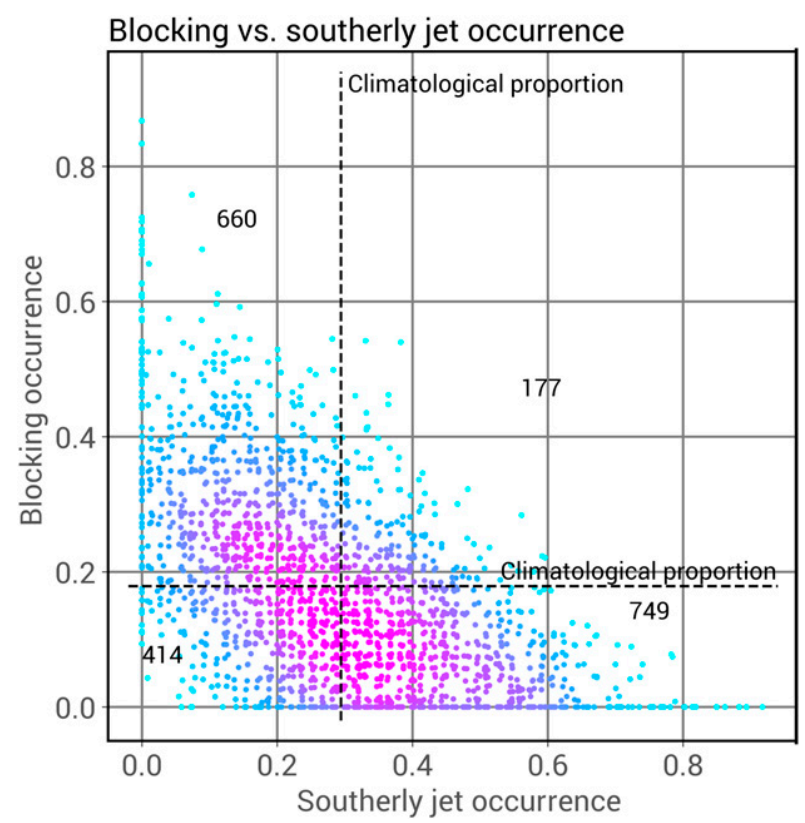

FIG. 7. Proportion of EC-Earth summers in the blocking region vs the southerly jet region of the phase space as defined in Fig. 5. The color indicates the density of points to improve the interpretability, with pink colors indicating higher density. The printed numbers indicate the total number of summers in each quadrant formed by the dashed lines representing the climatological probability. A small random noise is added to avoid overlapping points.

distinct in comparison to the southerly jet summer group, but this is partially because the southerly jet region covers a larger part (66 states vs 42 states in the blocking region) of the phase space.
To study the slow-moving underlying dynamics in both summer groups in more detail, we calculated the weighted average direction of 10-day transitions over the phase space in each state. We do this by splitting every 10-day transition from a specific state in a horizontal and vertical component and multiplying it by the corresponding transition probability, resulting in the weighted average horizontal and vertical direction of transitions starting in a specific state. In a similar manner, we calculated the weighted average absolute distance over the phase space of these transitions as an inverse persistence measure. Figure 9 displays both quantities with the directions given by the streamlines and the distance (inverse persistence) of 10-day transitions given by the coloring of the streamlines, with a darker color indicating a shorter distance (higher persistence). We see that the underlying dynamics in both groups differ significantly. The preferred regions are clearly distinguishable by following the direction of the arrows; during southerly jet summers, the streamlines converge to the southerly jet region, while for blocking summers the streamlines converge toward the blocking region. Once in their preferred state, summer trajectories also tend to stay there, as indicated by the long persistence (darker color shading of the arrows). In contrast to this, we see that summers in the southerly jet group can still reach the blocking region, but once they do, they tend to move out of these regions relatively quickly, as indicated by the short persistence (light shading of the arrows) in the blocking region in Fig. 9a. Likewise for the blocking summer group (Fig. 9b), summer trajectories still traverse the southerly jet region but also move out of this region relatively quickly. Figure 9 thus shows that the dynamics of the two groups of summers are fundamentally different, supporting the hypothesis that multiple attractors exist making it a fully intransitive system. It also establishes

\section{(a) Southerly jet summers Probability distribution over states}

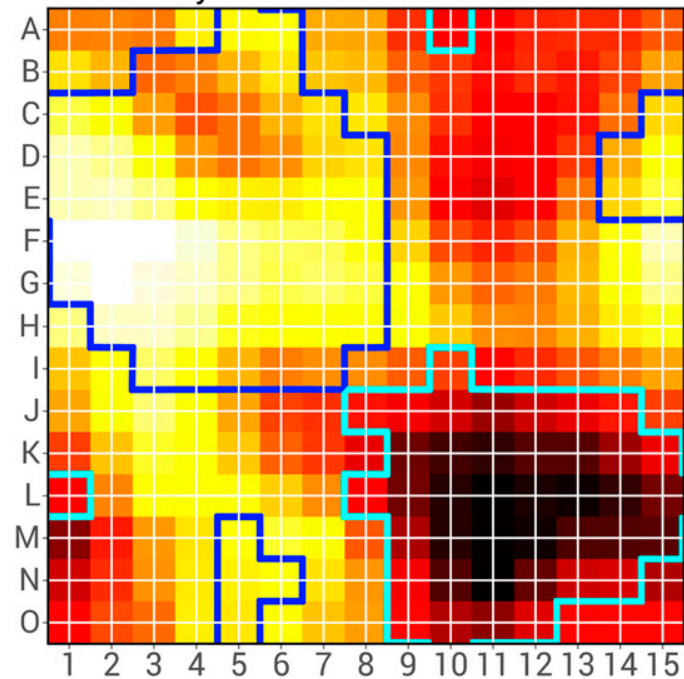

(b) Blocking summers Probability distribution over states

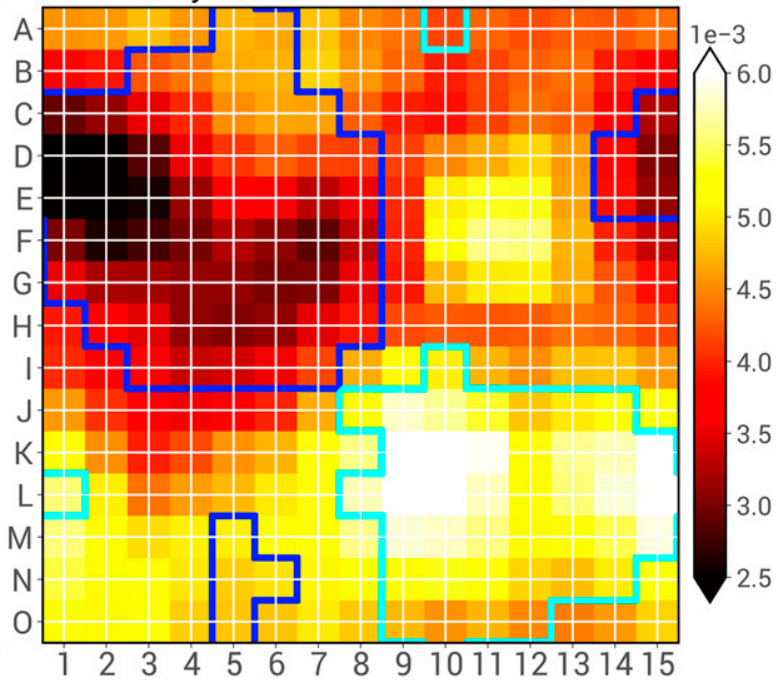

FIG. 8. Probability distribution over states in the (a) southerly jet summers and the (b) blocking summers, smoothed with a $3 \times 3$ filter. Lighter colors refer to relatively many days in this state and dark colors to relatively few days. Values sum up to 1 in both panels. Outlined regions indicate the southerly jet (dark blue) and blocking (cyan) region. 
(a) Southerly jet summers 10-day transition tendencies

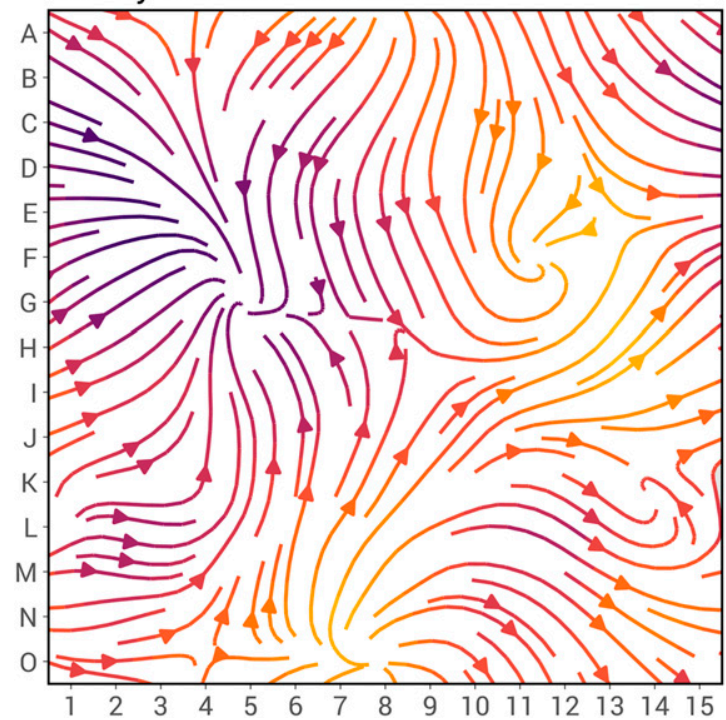

(b) Blocking summers 10-day transition tendencies

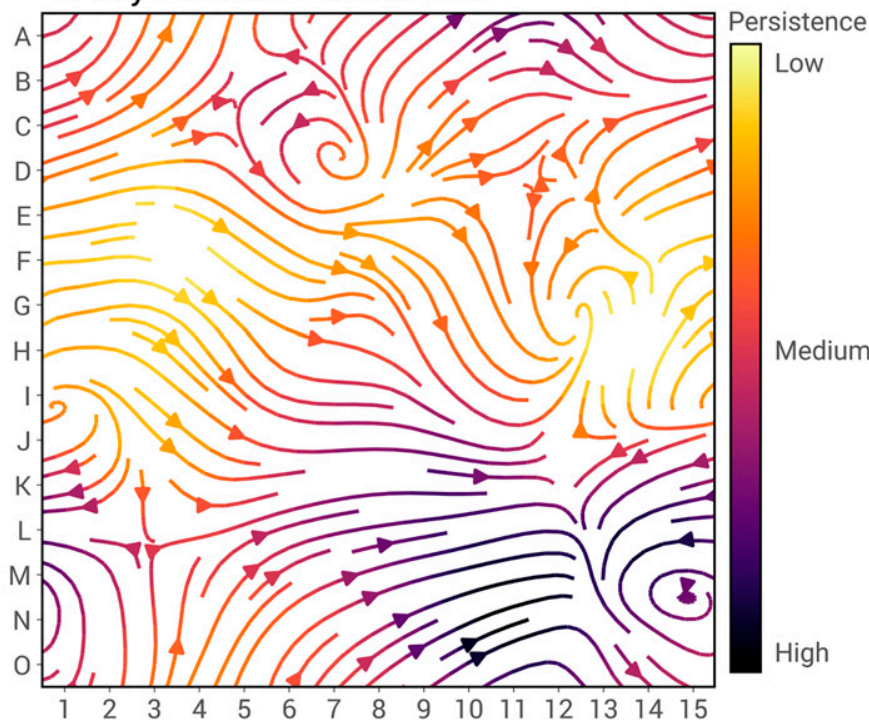

FIG. 9. Streamlines over the phase space for summers in (a) the southerly jet group and (b) the blocking group, depicting the tendencies of 10-day transitions through the phase space. Colors indicate the persistence of 10-day transitions. Darker colors indicate 10-day transitions stay relatively close (high persistence), and lighter colors indicate transitions jump farther away (low persistence).

that these contrasting regions of phase space do not strictly exclude each other but that the dynamical behavior is different: A summer governed by the blocking attractor can still see southerly jet days but tends to rapidly traverse through that part of phase space returning to the blocking region. The converse is true for summers governed by a southerly jet attractor.

\section{Discussion}

Persistent hot-dry or cold-wet summer weather can have significant impacts on agriculture, health, the energy sector, and the environment (Buras et al. 2020; Philip et al. 2020; Panteli and Mancarella 2015; Maracchi et al. 2005; Simmonds 2018). In this research, we studied jet stream dynamics behind these persistent summer extremes. Our study is motivated by work of Edward Lorenz, who postulated that summer circulation may be either fully or almost intransitive (Lorenz 1990). An intransitive atmosphere implies that part of the phase space (capturing circulation variability) cannot be reached within a specific summer. A fully intransitive atmosphere has multiple distinctly different attractors, each with their own preferred states and trajectories through phase space. In contrast, in an almost intransitive atmosphere each summer's trajectory is governed by the same attractor, but depending on the initial state some parts of phase space are unreachable within a single summer, simply because the summer is too short to traverse the complete phase space (Lorenz 1976). Intransitivity would have major implications for the predictability of summer weather, our understanding of the drivers of interannual variability of summer weather, and potentially climate risks associated with future circulation changes.
In this study, we tested the two Lorenz hypotheses for European summers, motivated by the persistent hot-dry summer of 2018 (Buras et al. 2020; Kornhuber et al. 2019). We analyze the trajectories over the phase space of circulation patterns and found in our first experiment that the memory of the initial state is on the order of a month, which is relatively long as compared to the duration of the "high-summer" period July-August. Moreover, the two initial states with the longest memory, a southerly shifted zonal jet stream and a blocking circulation, exclude each other to a high degree within a single summer season. This indicates that if a summer starts in either of these two states, this already gives a good indication of the typical circulation patterns for the remainder of that summer, thereby supporting the idea of almost intransitivity. Building on these results, in our second experiment we identified two groups of summers that differ in their climatological probability of individual states as well as their trajectories (i.e., the temporal evolution through phase space). The attractors do not restrict the summer trajectories to a specific part of the phase space, but the dynamical characteristics of the trajectories are different, leading to the emergence of preferred states. Thus, summers governed by the blocking attractor can reach the southerly jet region of the phase space, but when they do, they rapidly traverse through this region and return to their preferred blocked state. Likewise, summers governed by the southerly jet attractor can reach blocked states but will rapidly return to their preferred southerly jet state. The pronounced distinction between the dynamics of the two groups of summers, as given by their different trajectories, supports the hypothesis that there are multiple summer attractors governing the circulation dynamics (i.e., full intransitivity). 
Taken together, we presented substantial evidence in favor of the hypothesis that intransitivity is a fundamental property of the atmosphere in summer. Our results suggest that the summer atmosphere displays characteristics of both almost intransitivity and full intransitivity. However, the findings in our research should be treated with caution given the underlying assumptions.

First, one could question whether the two groups we found in the second experiment truly represent two different attractors. Potentially, the different dynamical characteristics we identified could result from the way we initialized the groups, since this was based on the occurrence of southerly jet states and blocking states, respectively. This might bias the algorithm in such a way that the transition matrices of the two groups favor trajectories that stay close to the group's respective most frequently traversed region (i.e., either the blocking region or the southerly jet region). Trajectories that return to these regions by coincidence could therefore be interpreted by the algorithm as being governed by fundamentally different dynamics. We cannot fully rule out whether such bias may have a substantial influence and thus we have not explicitly shown that the atmospheric circulation is fully intransitive. However, we provide a counterexample against the assumption that all summers are governed by a single attractor. Thereby we fulfil a minimum requirement for the existence of full intransitivity.

Additionally, there might be more attractors with their own distinctive dynamical characteristics than the two we identified in the second experiment. An indication for this is the large group of EC-Earth summers (20.7\%; see Fig. 7) that have a low presence in both the southerly jet region and blocking region. These summers are now roughly evenly divided over the southerly jet summer group and the blocking summer group (216 in the southerly jet summer group versus 198 in the blocking summer group). In principle, these summers might have their own underlying dynamics, characterized by a low persistence in both regions for example. However, for full intransitivity to occur two attractors are sufficient, and thus for hypothesis testing it is sufficient to only identify two distinct sets of dynamics. In other words, the potential existence of more than two attractors would not disqualify the general conclusion of this study. While the exact number of circulation regimes is still an ongoing debate (Ghil 2019), the presence of two regimes (one with blocked flow and one with zonal flow and variations thereof) is most established in the literature (Lorenz 1990; Charney and DeVore 1979; Charney et al. 1981; Hannachi et al. 2017; Branstator and Berner 2005; Sempf et al. 2007; Tantet et al. 2015).

A third limitation of our study is the use of simulated climate data to have a sufficient sample size. On relatively short synoptic time scales, persistence in climate models is likely overestimated due to an underestimation of the local dimensionality (i.e., the number of directions in phase space to which the system can evolve for a given state; Faranda et al. 2017). This lower local dimensionality implies that the model is more likely to remain in the same state. On the other hand, there is considerable evidence that climate models tend to underestimate long-lived events (beyond a week) in the midlatitudes such as persistent blocking, especially over Europe in summer (Schiemann et al. 2020; Davini and D'Andrea 2016). These differences raise questions as to what extend the results can be generalized to the real atmosphere. This will be the subject of future work.

Here we have not studied the underlying physical processes that lead to the long memory (the first experiment) or the existence of two underlying attractors (the second experiment). While we analyzed atmospheric fields, the slowly varying components of the Earth system, such as sea surface temperature, soil moisture levels, or snow cover, likely play an important role (Lorenz 1976, 1990; Kingston et al. 2015; Ionita et al. 2012; Fischer et al. 2007; García-Herrera et al. 2010; Della-Marta et al. 2007). We have shown that the initial state influences which parts of the phase space a summer is likely to visit (i.e., almost intransitivity), but it is likely that anomalies in the boundary conditions of the atmosphere continue to influence the circulation dynamics during summer as well, since they add a longer-term memory component to the system. Following Lorenz (1976), we suspect interactions between the boundary conditions and the atmosphere may drive the circulation into a specific regime of preferred trajectories (i.e., full intransitivity), for example through soil-moisture feedbacks (Boé 2013; Fischer et al. 2007) or oceanatmosphere interactions (Ossó et al. 2018; Sutton and Hodson 2005; Dong et al. 2013). Since we used data from a fully coupled climate model these processes are in principle represented and thus accounted for in our analyses. Future work should look into the role of these boundary conditions versus the role of internal atmospheric dynamics in causing intransitive dynamics. Potentially, one might also be able to identify different spring precursors for the two different summer attractors. If the specific summer attractor and/or the initial circulation state can be identified at an early stage, this may provide insight into whether the rest of a summer will be mostly hot-dry or cold-wet (Ionita et al. 2017; Screen 2013; Dong et al. 2013; Sutton and Hodson 2005). This might provide predictive information if the initial circulation state is either a southerly jet or a blocking state, as those states are most persistent and likely governed by different attractors. Though speculative at this stage, this might provide a window of opportunity to forecast European summer weather at subseasonal time scales (Mariotti et al. 2020). Moreover, southerly jet or blocking states are linked to weather conditions that can significantly impact societies or ecosystems and thus early warnings could have strong benefits (e.g., Vogel et al. 2019; Buras et al. 2020; Philip et al. 2020; Maracchi et al. 2005). Future work should identify possible precursors in the slow components of the climate system and their interaction with the atmosphere (Quesada et al. 2012). Finally, an important question is how circulation is likely to change in a future warmer world (Hannachi et al. 2017). Our SOM phase space and trajectory analyses, as presented here, may provide a powerful tool to understand and quantify future regional circulation changes.

This study's aim was purely to enhance fundamental understanding of the dynamics of the atmospheric circulation, motivated by its implications for predictability and potential climate risks. Our results solidify the framework postulated by Lorenz 


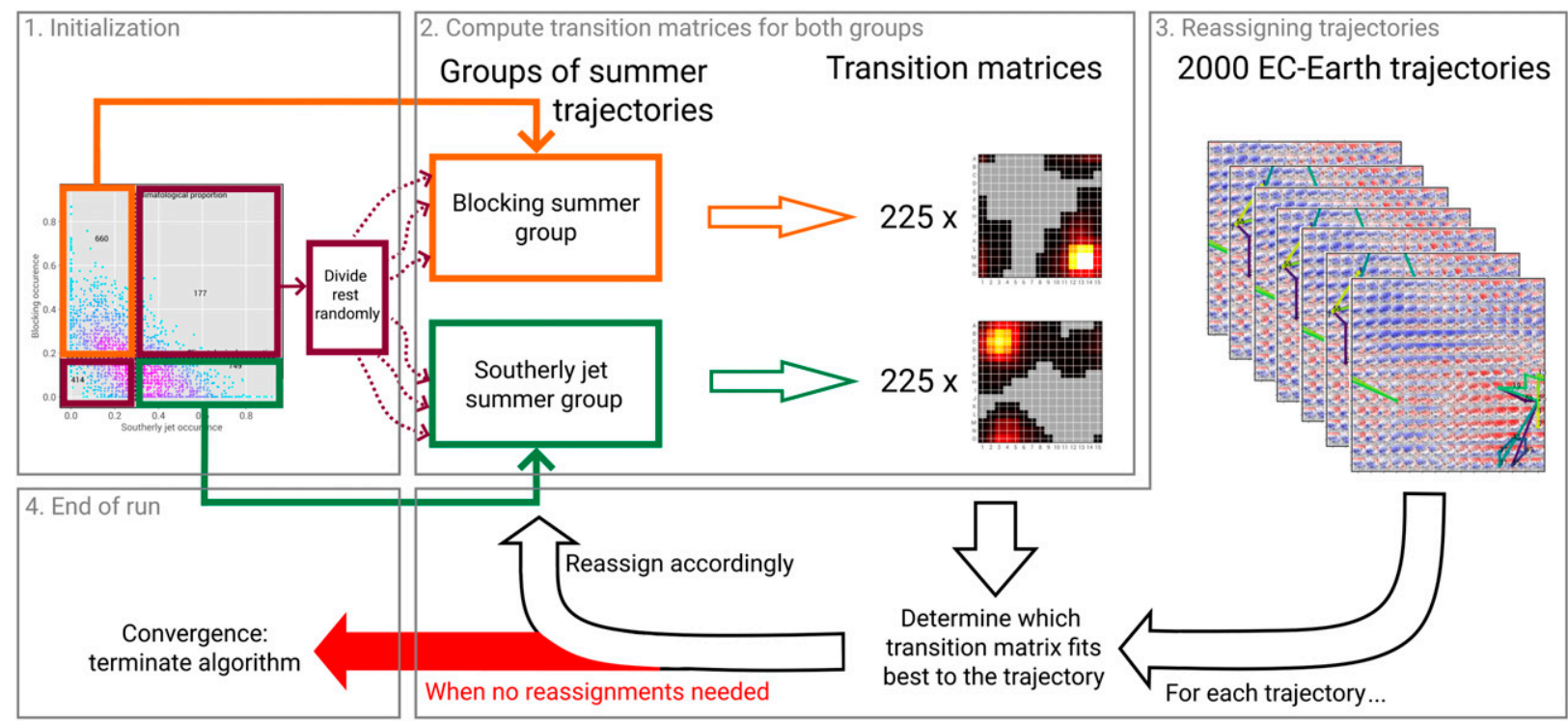

FIG. A1. Schematic representation of the custom clustering method used to find two sets of summer trajectories through phase space that have distinctly different corresponding transition matrices. A detailed explanation is available in the appendix.

that, next to chaotic behavior, also intransitivity can be a fundamental property of the atmosphere and that, if it is, it is likely so in summer. This concept merits strong consideration as a potential cause of interannual variability in summer conditions.

Acknowledgments. The EC-Earth experiments were generated in the HiWAVES3 project, for which funding was supplied by the Netherlands Organisation of Scientific Research (NWO) under Grant ALWCL.2016.2. The authors thank three anonymous reviewers for their constructive comments, which helped to improve the manuscript. DC and FS acknowledge support from the Netherlands Organisation for Scientific Research (NWO), Grant 016.Vidi.171.011.

Data availability statement. EC-Earth data are available from the corresponding author upon reasonable request.

\section{APPENDIX}

\section{Detailed Description of Custom Trajectory Clustering Method}

Similar to $k$-means, the algorithm we developed is iterative and needs two initial groups of summers. However, instead of computing the distance between an observation and a cluster mean, our algorithm computes how well a trajectory (the observation) fits a cluster's transition matrix (capturing the general dynamics of the cluster). Based on our results from the Markov experiment, we chose to make the initial grouping not completely random. Since the blocking and southerly jet region exclude each other to a high degree, it follows that summers in these regions may also exhibit different dynamics, being persistent in their preferred region and breaking out of their contrasting region relatively quickly. Therefore, we define the initial groups based on the EC-Earth summers' presence in the blocking and southerly jet region (step 1 in Fig. A1). All summers that have an above climatological presence in the blocking region and a below climatological presence in the southerly jet region-summers that fall in the top-left quadrant of Fig. 7-are assigned to the "blocking summers" group. Likewise, all summers that fall in the bottom-right quadrant of Fig. 7 are assigned to the "southerly jet summers" group. Summer trajectories in the other two quadrants are divided randomly over the two groups, hence the initial grouping is different in each run of the clustering algorithm. Then, the three-day-ahead transition matrices (e.g., Fig. 4) for the ECEarth summers in both groups are computed, resulting in two transition matrices (step 2). We use the 3-day-ahead transition probabilities since differences in 3-day transition matrices are more distinguishable than differences in 1-day transition matrices. This is because processes with a frequency of three days or larger are filtered out of the data.

One iteration of the clustering algorithm consists of reassigning each summer trajectory to their most representative group in terms of their dynamics (step 3 in Fig. A1), and recomputing the transition matrices for both groups of summers once the reassignment of all 2000 EC-Earth summers is finished (step 2 in Fig. A1). Reassignment of a summer trajectory to another group is based on how high the groups score for that trajectory. The scores given to both groups for an EC-Earth summer trajectory are computed by comparing the three-dayahead transition probabilities between groups. For each 3-day transition in a summer trajectory, the group with the transition matrix with the highest corresponding likelihood of that transition (i.e., highest transition probability) receives a point. Since summer trajectories are 62 days long (July-August), each summer consists of $62-3=59$ three-day transitions. The sum of the scores of both groups for any summer trajectory is always 
equal to 59, since one transition results in either a point for the blocking summer group or for the southerly jet summer group. The summer trajectory is then assigned to the group with the highest score, since this group's underlying dynamics are most representative for that trajectory.

When all summer trajectories are reassigned, the three-dayahead transition matrices for both groups of summers are recomputed (step 2 in Fig. A1). This process (steps 2 and 3 ) is repeated until the summer trajectories do not switch groups anymore (step 4). The interplay between the varying group compositions and the varying transition matrices ensures that the transition matrix in each group converges to its own characteristic dynamics. It also ensures that every summer is more likely to behave according to the underlying dynamics (i.e., transition matrix) of the group it is assigned to, rather than the dynamics of the alternative group, since the score for the alternative group is always lower.

\section{REFERENCES}

Beillouin, D., B. Schauberger, A. Bastos, P. Ciais, and D. Makowski, 2020: Impact of extreme weather conditions on European crop production in 2018. Philos. Trans. Roy. Soc., 375B, 20190 510, https://doi.org/10.1098/rstb.2019.0510.

Boé, J., 2013: Modulation of soil moisture-precipitation interactions over France by large scale circulation. Climate Dyn., 40, 875-892, https://doi.org/10.1007/s00382-012-1380-6.

Branstator, G., and J. Berner, 2005: Linear and nonlinear signatures in the planetary wave dynamics of an AGCM: Phase space tendencies. J. Atmos. Sci., 62, 1792-1811, https://doi.org/ 10.1175/JAS3429.1.

Buras, A., A. Rammig, and C. S. Zang, 2020: Quantifying impacts of the 2018 drought on European ecosystems in comparison to 2003. Biogeosciences, 17, 1655-1672, https://doi.org/10.5194/ bg-17-1655-2020.

Cassou, C., L. Terray, and A. S. Phillips, 2005: Tropical Atlantic influence on European heat waves. J. Climate, 18, 2805-2811, https://doi.org/10.1175/JCLI3506.1.

Charney, J. G., and J. G. DeVore, 1979: Multiple flow equilibria in the atmosphere and blocking. J. Atmos. Sci., 36, 12051216, https://doi.org/10.1175/1520-0469(1979)036<1205: MFEITA $>2.0 . \mathrm{CO} ; 2$.

_ J. Shukla, and K. Mo, 1981: Comparison of a barotropic blocking theory with observation. J. Atmos. Sci., 38, 762-779, https:// doi.org/10.1175/1520-0469(1981)038<0762:COABBT >2.0.CO;2.

Davini, P., and F. D'Andrea, 2016: Northern Hemisphere atmospheric blocking representation in global climate models: Twenty years of improvements? J. Climate, 29, 8823-8840, https://doi.org/10.1175/JCLI-D-16-0242.1.

Della-Marta, P. M., J. Luterbacher, H. von Weissenfluh, E. Xoplaki, M. Brunet, and H. Wanner, 2007: Summer heat waves over western Europe 1880-2003, their relationship to large-scale forcings and predictability. Climate Dyn., 29, 251275, https://doi.org/10.1007/s00382-007-0233-1.

Dong, B., R. Sutton, and T. Woollings, 2013: The extreme European summer 2012 [in "Explaining Extreme Events of 2012 from a Climate Perspective"]. Bull. Amer. Meteor. Soc., 94 (9), S28-S32, https://doi.org/10.1175/BAMS-D-13-00085.1.

Drouard, M., K. Kornhuber, and T. Woollings, 2019: Disentangling dynamic contributions to summer 2018 anomalous weather over Europe. Geophys. Res. Lett., 46, 12 537-12 546, https:// doi.org/10.1029/2019GL084601.
Faranda, D., G. Messori, and P. Yiou, 2017: Dynamical proxies of North Atlantic predictability and extremes. Sci. Rep., 7, 41278, https://doi.org/10.1038/srep41278.

Fischer, E. M., S. I. Seneviratne, P. L. Vidale, D. Lüthi, and C. Schär, 2007: Soil moisture-atmosphere interactions during the 2003 European summer heat wave. J. Climate, 20, 50815099, https://doi.org/10.1175/JCLI4288.1.

Frame, T. H., M. H. Ambaum, S. Gray, and J. Methven, 2011: Ensemble prediction of transitions of the North Atlantic eddydriven jet. Quart. J. Roy. Meteor. Soc., 137, 1288-1297, https:// doi.org/10.1002/qj.829.

Franzke, C., and T. Woollings, 2011: On the persistence and predictability properties of North Atlantic climate variability. J. Climate, 24, 466-472, https://doi.org/10.1175/2010JCLI3739.1.

- , and Coauthors, 2020: The structure of climate variability across scales. Rev. Geophys., 58, e2019RG000657, https:// doi.org/10.1029/2019RG000657.

García-Herrera, R., J. Díaz, R. M. Trigo, J. Luterbacher, and E. M. Fischer, 2010: A review of the European summer heat wave of 2003. Crit. Rev. Environ. Sci. Technol., 40, 267-306, https:// doi.org/10.1080/10643380802238137.

Ghil, M., 2019: A century of nonlinearity in the geosciences. Earth Space Sci., 6, 1007-1042, https://doi.org/10.1029/2019EA000599.

Hannachi, A., D. M. Straus, C. L. Franzke, S. Corti, and T. Woollings, 2017: Low-frequency nonlinearity and regime behavior in the Northern Hemisphere extratropical atmosphere. Rev. Geophys., 55, 199-234, https://doi.org/10.1002/2015RG000509.

Hazeleger, W., and Coauthors, 2012: EC-Earth V2.2: Description and validation of a new seamless Earth system prediction model. Climate Dyn., 39, 2611-2629, https://doi.org/10.1007/ s00382-011-1228-5.

Ionita, M., G. Lohmann, N. Rimbu, S. Chelcea, and M. Dima, 2012: Interannual to decadal summer drought variability over Europe and its relationship to global sea surface temperature. Climate Dyn., 38, 363-377, https://doi.org/10.1007/s00382-011-1028-y.

— , and Coauthors, 2017: The European 2015 drought from a climatological perspective. Hydrol. Earth Syst. Sci., 21, 13971419, https://doi.org/10.5194/hess-21-1397-2017.

Jain, A. K., 2010: Data clustering: 50 years beyond K-means. Pattern Recognit. Lett., 31, 651-666, https://doi.org/10.1016/ j.patrec.2009.09.011.

Kingston, D. G., J. H. Stagge, L. M. Tallaksen, and D. M. Hannah, 2015: European-scale drought: Understanding connections between atmospheric circulation and meteorological drought indices. J. Climate, 28, 505-516, https://doi.org/10.1175/JCLID-14-00001.1.

Kohonen, T., 1990: The self-organizing map. Proc. IEEE, 78, 14641480, https://doi.org/10.1109/5.58325.

Kolmogorov, A., 1933: Sulla determinazione empirica di una lgge di distribuzione. Inst. Ital. Attuari. Giorn., 4, 83-91.

Kornhuber, K., S. Osprey, D. Coumou, S. Petri, V. Petoukhov, S. Rahmstorf, and L. Gray, 2019: Extreme weather events in early summer 2018 connected by a recurrent hemispheric wave-7 pattern. Environ. Res. Lett., 14, 054002, https://doi.org/ 10.1088/1748-9326/ab13bf.

Leith, C., 1973: The standard error of time-average estimates of climatic means. J. Appl. Meteor., 12, 1066-1069, https://doi.org/ 10.1175/1520-0450(1973)012<1066:TSEOTA > 2.0.CO;2.

Li, M., Y. Yao, I. Simmonds, D. Luo, L. Zhong, and X. Chen, 2020: Collaborative impact of the NAO and atmospheric blocking on European heatwaves, with a focus on the hot summer of 2018. Environ. Res. Lett., 15, 114003, https://doi.org/10.1088/ 1748-9326/aba6ad. 
Lorenz, E. N., 1963: Deterministic nonperiodic flow. J. Atmos. Sci., 20, 130-141, https://doi.org/10.1175/1520-0469(1963)020<0130: $\mathrm{DNF}>2.0 . \mathrm{CO} ; 2$.

- 1968: Climatic determinism. Causes of Climatic Change, Springer, 1-3, https://doi.org/10.1007/978-1-935704-38-6_1.

- 1976: Nondeterministic theories of climatic change. Quat. Res., 6, 495-506, https://doi.org/10.1016/0033-5894(76)90022-3.

, 1982: Atmospheric predictability experiments with a large numerical model. Tellus, 34, 505-513, https://doi.org/10.3402/ tellusa.v34i6.10836.

, 1990: Can chaos and intransitivity lead to interannual variability? Tellus, 42A, 378-389, https://doi.org/10.3402/tellusa.v42i3.11884.

Lu, J., and T. Schneider, 2017: Evolving perspectives on abrupt seasonal changes of the general circulation. Adv. Atmos. Sci., 34, 1185-1194, https://doi.org/10.1007/s00376-017-7068-4.

Maracchi, G., O. Sirotenko, and M. Bindi, 2005: Impacts of present and future climate variability on agriculture and forestry in the temperate regions: Europe. Climatic Change, 70, 117-135, https://doi.org/10.1007/s10584-005-5939-7.

Mariotti, A., and Coauthors, 2020: Windows of opportunity for skillful forecasts subseasonal to seasonal and beyond. Bull. Amer. Meteor. Soc., 101, E608-E625, https://doi.org/10.1175/ BAMS-D-18-0326.1.

Ohba, M., S. Kadokura, and D. Nohara, 2016: Impacts of synoptic circulation patterns on wind power ramp events in East Japan. Renew. Energy, 96, 591-602, https://doi.org/10.1016/ j.renene.2016.05.032.

Ossó, A., R. Sutton, L. Shaffrey, and B. Dong, 2018: Observational evidence of European summer weather patterns predictable from spring. Proc. Natl. Acad. Sci. USA, 115, 59-63, https:// doi.org/10.1073/pnas.1713146114.

Panteli, M., and P. Mancarella, 2015: Influence of extreme weather and climate change on the resilience of power systems: Impacts and possible mitigation strategies. Electr. Power Syst. Res., 127, 259-270, https://doi.org/10.1016/j.epsr.2015.06.012.

Pasquier, J., S. Pfahl, and C. M. Grams, 2019: Modulation of atmospheric river occurrence and associated precipitation extremes in the North Atlantic region by European weather regimes. Geophys. Res. Lett., 46, 1014-1023, https://doi.org/ 10.1029/2018GL081194.

Pfleiderer, P., C.-F. Schleussner, K. Kornhuber, and D. Coumou, 2019: Summer weather becomes more persistent in a $2^{\circ} \mathrm{C}$ world. Nat. Climate Change, 9, 666-671, https://doi.org/10.1038/s41558019-0555-0.

Philip, S. Y., S. F. Kew, K. van der Wiel, N. Wanders, and G. J. van Oldenborgh, 2020: Regional differentiation in climate change induced drought trends in the Netherlands. Environ. Res. Lett., 15, 094 081, https://doi.org/10.1088/1748-9326/ab97ca.

Quesada, B., R. Vautard, P. Yiou, M. Hirschi, and S. I. Seneviratne, 2012: Asymmetric European summer heat predictability from wet and dry southern winters and springs. Nat. Climate Change, 2, 736-741, https://doi.org/10.1038/nclimate1536.

Ross, D. A., J. Lim, R.-S. Lin, and M.-H. Yang, 2008: Incremental learning for robust visual tracking. Int. J. Comput. Vis., 77, 125-141, https://doi.org/10.1007/s11263-007-0075-7.

Schiemann, R., and Coauthors, 2020: Northern Hemisphere blocking simulation in current climate models: Evaluating progress from the Climate Model Intercomparison Project phase 5 to 6 and sensitivity to resolution. Wea. Climate Dyn., 1, 277-292, https://doi.org/10.5194/wcd-1-277-2020.

Screen, J. A., 2013: Influence of Arctic sea ice on European summer precipitation. Environ. Res. Lett., 8, 044015, https:// doi.org/10.1088/1748-9326/8/4/044015.

Sempf, M., K. Dethloff, D. Handorf, and M. V. Kurgansky, 2007: Toward understanding the dynamical origin of atmospheric regime behavior in a baroclinic model. J. Atmos. Sci., 64, 887904, https://doi.org/10.1175/JAS3862.1.

Simmonds, I., 2018: What causes extreme hot days in Europe? Environ. Res. Lett., 13, 071 001, https://doi.org/10.1088/17489326/aacc78.

Sutton, R. T., and D. L. Hodson, 2005: Atlantic Ocean forcing of North American and European summer climate. Science, 309, 115-118, https://doi.org/10.1126/science.1109496.

Tantet, A., F. R. van der Burgt, and H. A. Dijkstra, 2015: An early warning indicator for atmospheric blocking events using transfer operators. Chaos, 25, 036406, https://doi.org/10.1063/ 1.4908174.

Vanderkelen, I., and Coauthors, 2020: A novel method for assessing climate change impacts in ecotron experiments. Int. J. Biometeor., 64, 1709-1727, https://doi.org/10.1007/s00484020-01951-8.

van der Wiel, K., N. Wanders, F. Selten, and M. Bierkens, 2019: Added value of large ensemble simulations for assessing extreme river discharge in a $2^{\circ} \mathrm{C}$ warmer world. Geophys. Res. Lett., 46, 2093-2102, https://doi.org/10.1029/2019GL081967.

Vogel, M. M., J. Zscheischler, R. Wartenburger, D. Dee, and S. I. Seneviratne, 2019: Concurrent 2018 hot extremes across the Northern Hemisphere due to human-induced climate change. Earth's Future, 7, 692-703, https://doi.org/10.1029/2019EF001189.

Yeh, T.-C., 1959: The abrupt change of circulation over the Northern Hemisphere during June and October. The Atmosphere and the Sea in Motion, B. Bolin, Ed., Oxford University Press, 249-267. 